\title{
PAIRS OF GALAXIES IN LOW DENSITY REGIONS
}

\section{OF A COMBINED REDSHIFT CATALOG}

\author{
Jane C. Charlton (University of Arizona) \\ Edwin E. Salpeter (Cornell University)
}

\begin{abstract}
The distributions of projected separations and radial velocity differences of pairs of galaxies in the CfA and SSRS redshift catalogs are examined. We focus on pairs that fall in low density environments rather than in clusters or large groups. The projected separation distribution is nearly flat, while uncorrelated galaxies would have given one linearly rising with $r_{p}$. There is no break in this curve even below $50 \mathrm{kpc}$, the minimum halo size consistent with measured galaxy rotation curves. The significant number of pairs at small separations is inconsistent with the N-body result that galaxies with overlapping halos will rapidly merge, unless there are significant amounts of matter distributed out to a few hundred kpc of the galaxies. This dark matter may either be in distinct halos or more loosely distributed. Large halos would allow pairs at initially large separations to head toward merger, replenishing the distribution at small separations. In the context of this model, we estimate that roughly $10-25 \%$ of these low density galaxies are the product of a merger, compared with the elliptical / S0 fraction of $18 \%$, observed in low density regions of the sample.
\end{abstract}

\section{Introduction}

The classical samples of binary galaxies (e.g., those of Turner (1976) and Kharachentsev (1974)) included pairs on the basis of their projected separation, but the availability of redshift catalogs now allows the incorporation of radial velocities in a pair selection criterion. We examine the distributions of radial velocity difference $\Delta v$ and projected separation $r_{p}$ of pairs in the Northern CfA 14.5 magnitude limited, and the comparable Southern Sky Redshift Survey diameter limited, redshift catalogs. This study is similar in style to those of Rivolo and Yahil (1981) and of Davis and Peebles (1983). In order to reduce the degree of contamination by pairs that are members of triplets or larger groups, it is common to apply an isolation criterion (e.g., Turner 1979; Rivolo and Yahil 1981). We do not apply an isolation criterion, so as to avoid any subtle selection biases it might introduce, but instead use an overall density criterion to separately consider pairs in low and high density regions. In this paper, we focus on pairs residing in low density regions which may include members of small groups, as well as isolated binaries. The $\Delta v$ and $r_{p}$ histograms in conjunction with previous $\mathrm{N}$-body results will allow us to draw conclusions about the sizes of galactic halos. Finally, a revival of the replenishment picture of galaxy mergers (Ostriker and Turner 1979) will allow a rough estimate of the galaxy merger rate.

\section{The Redshift Samples}

We consider a combined pair sample extracted from the Northern CfA and the Southern Sky Redshift Survey catalogs (SSRS). The Northern region $(\delta \geq 0, b \geq 40 \mathrm{deg})$ of 
the CfA redshift survey (Huchra and Geller 1982), complete to magnitude 14.5, contains $\sim 1900$ galaxies. Many now have $21 \mathrm{~cm}$ redshift measurements so that the median error in the radial velocity, $v$, is $23 \mathrm{~km} / \mathrm{s}$. The SSRS region $(\delta \leq-17.5 \mathrm{deg}, b \leq-30 \mathrm{deg})$ (da Costa, et. al. 1988$)$ is roughly comparable in area ( $\sim 1.75$ steradians) and contains roughly the same number of galaxies since its diameter limit $\left(\leq 1.26^{\prime}\right)$ is comparable to the Northern magnitude limit. We compute the selection function (which decreases by a factor of $\sim 10$ over the range $1100<v<4500 \mathrm{~km} / \mathrm{s}$ in these catalogs), and use it to compensate the observed density at different $v$. (Fortunately this does not bias the $r_{p}$ distribution, since pairs with larger $r_{p}$ are equally likely to be excluded.)

We classify each galaxy as being in a high or low density environment by counting the number of neighbors within $r_{p}<5 \mathrm{Mpc}$ (using $h=0.75$ ) and $\Delta v<375 \mathrm{~km} / \mathrm{s}$ of each galaxy and, using the selection function, scaling this count to the number that would be expected if the primary galaxy were at $v=1100 \mathrm{~km} / \mathrm{s}$. (This volume, although it is much larger than small groups and cluster cores, is smaller than a supercluster.) This procedure yields a median body-centered density of $\sim 0.06$ galaxies / $\mathrm{Mpc}^{3}$ brighter than absolute magnitude $M=-15.7+5 \log h$. Galaxies with densities lower than the median are considered to be in a low density region, and are very seldom members of known clusters such as Virgo, Eridanus, or Fornax. The low density galaxies have a median density of $\sim 0.03$ galaxies $/ \mathrm{Mpc}^{3}$, while the corresponding number for high density is 0.1 galaxies / $\mathrm{Mpc}^{3}$.

\section{Radial Velocity Difference and Projected Separation Distributions}

Radial velocity difference histograms for pairs with $r_{p}$ in various ranges are given in Figures 1a-d. Only pairs with the primary member in a low density environment are included in the histograms. A primary galaxy must be at least as far from the catalog edges as the maximum $\Delta v$ or $r_{p}$ of pairs displayed in the histogram so that catalog edges do not lead to bias in the distribution. The full sample (solid line) includes "isolated pairs" as well as "group pairs", and "deceptive velocity difference pairs" (DVDP). In some cases the isolated pairs are the brightest two members of a distant group, but most of the group pairs do not contain both of the two brightest members. In "deceptive velocity difference pairs" the peculiar velocities of pair members (relative to smooth Hubble flow) are oriented so as to yield small values of $\Delta v$, although the true physical separation in the $z$ direction is quite large. These are to be distinguished from "optical pairs" that have small $r_{p}$, but large $\Delta v$. In Figure 1 we see that the level of the histogram at large $\Delta v$ increases with $r_{p}$, as would be expected due to the increased area of the bin. At all $r_{p}$ there is an excess, over the background, of pairs with small $\Delta v(<150 \mathrm{~km} / \mathrm{s})$; so we focus on these pairs and examine their $r_{p}$ distribution. Figure $2 \mathrm{a}$ gives the distribution of $r_{p}$ out to $3 \mathrm{Mpc}$, and Figure $2 \mathrm{~b}$ is a "blow-up" of the region at small $r_{p}(<0.4 \mathrm{Mpc})$ that is of particular interest. The distribution is remarkably flat, with equal numbers in equal $r_{p}$ bins, even down to $r_{p}$ values as low as $20 \mathrm{kpc}$. No significant kinks or breaks in the curve are apparent. (For larger $\Delta v$ pairs the slope of the $r_{p}$ distribution increases, with fewer pairs at small $r_{p}$.) We see that the phase space density of pairs is largest when both $r_{p}$ and $\Delta v$ are small. 


\section{"Halo" Sizes}

First, we should note that observed galaxy rotation curves demonstrate that a $\rho \sim$ $r^{-2}$ halo is present, in many instances, out to $50 \mathrm{kpc}$. Thus we know that pairs with separations $\leq 100 \mathrm{kpc}$ are likely to have overlapping halos. This is important because $\mathrm{N}$ body simulations (White 1978; Carlberg 1982) demonstrate that once halos overlap mergers occur readily (within $\sim$ one orbital period) due to dynamical friction. The observed flat, smooth, $r_{p}$ distribution is inconsistent with a picture in which halos truncate at small $r_{p}$, since rapid depletion of $r_{p}<2 R_{\text {halo }}$ pairs would occur while the $r_{p}$ distribution at larger
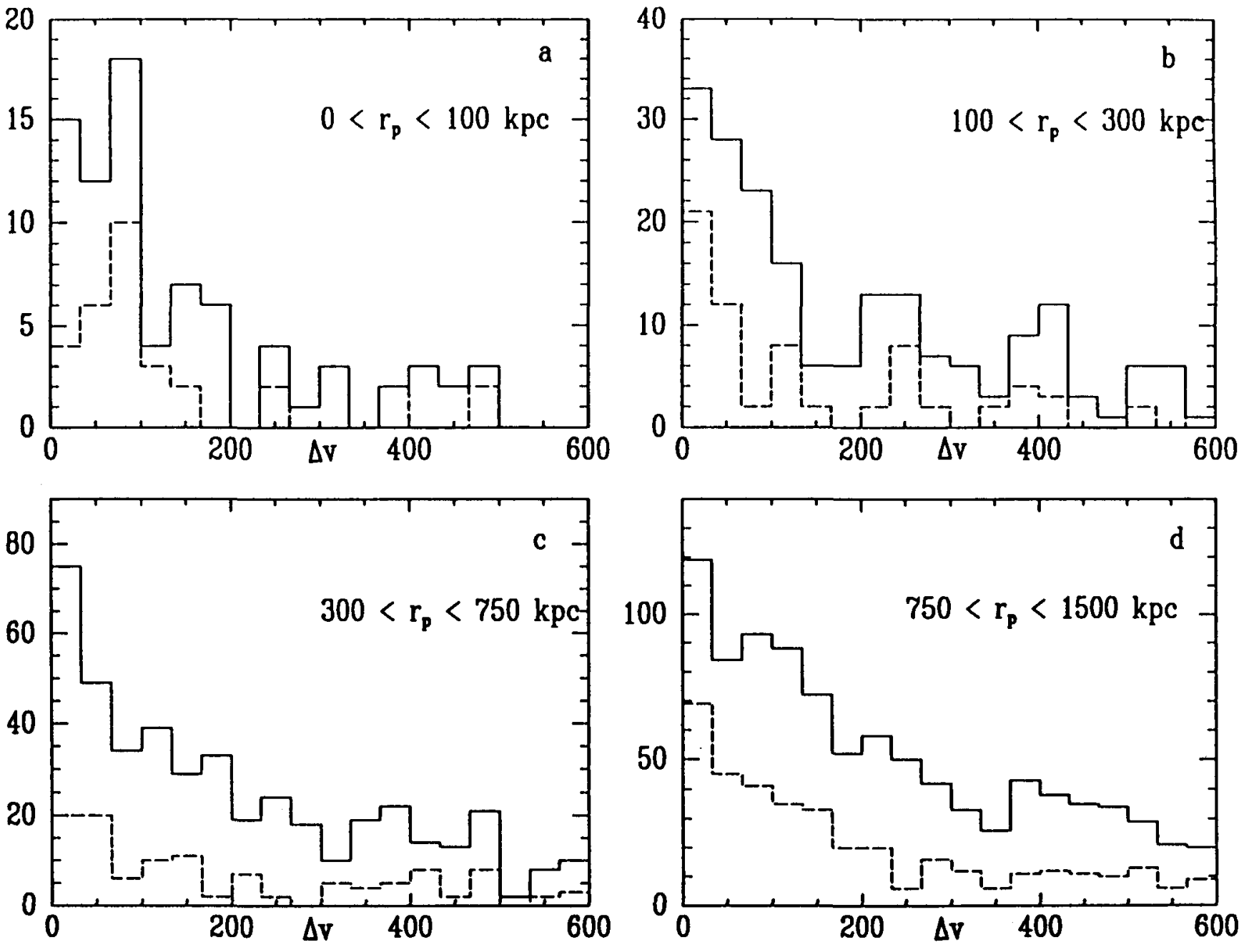

Figure 1: Histograms of the radial velocity differences $\Delta v$ of pairs of galaxies in low density environments, for four different ranges of pair projected separation $r_{p}$, are given. The units of $\Delta v$ on the horizontal axis are $\mathrm{km} / \mathrm{s}$. The solid curve represents all low density pairs, while the dotted curve is only those that are isolated within $300 \mathrm{~km} / \mathrm{s}$ in $\Delta v$ and $0.75 \mathrm{Mpc}$ in $r_{p}$. 
values would remain intact. Larger halos could preserve a flat $r_{p}$ distribution because the small $r_{p}$ region, depleted by rapid mergers, would be replenished by originally larger $r_{p}$ pairs headed toward merger (see Ostriker and Turner 1979). In a realistic model, halos would have a range of sizes, and orbits, a range of eccentricities, thus it is difficult to give a specific lower limit on halo sizes. For $r_{p}>$ several hundred kpc many pairs are likely to have orbital periods exceeding a Hubble time, thus complete depletion of smaller $r_{p}$ would not be expected. It is clear, however, that halos smaller than a few hundred $\mathrm{kpc}$ are inconsistent with the observed flat $r_{p}$ distribution.

We want to emphasize that we are using the term "halo" quite loosely. The above arguments cannot distinguish between a $\rho \sim r^{-2}$ halo cutting off abruptly at a few hundred $\mathrm{kpc}$, and one that has relatively less mass beyond $\sim 50 \mathrm{kpc}$, (e.g., $\rho \sim r^{-3}$ ). Alternatively, the dark matter could be evenly distributed on larger scales and loosely concentrated about pairs and groups of galaxies, rather than attached in the form of individual halos.

The above picture can be described by the parameter $\dot{R}$, the orbit averaged rate of change of a pairs' three dimensional separation. The shape of the $r_{p}$ distribution allows us to place loose constraints on the dependence of $\dot{R}$ on the separation $R$ of a pair. Roughly speaking, $\dot{R} \sim R^{\beta}$ where $-1 / 2 \leq \beta \leq 0$ would be consistent, since for $\beta=-1 / 2$ the increase in speed as the galaxies approach could be compensated in the $r_{p}$ distribution by projection effects. The simple picture in which the approach is at a constant rate, $\beta=0$, (shifting the $r_{p}$ distribution to the left with time) is also certainly allowed, and we shall estimate the galaxy merger rate in this context.
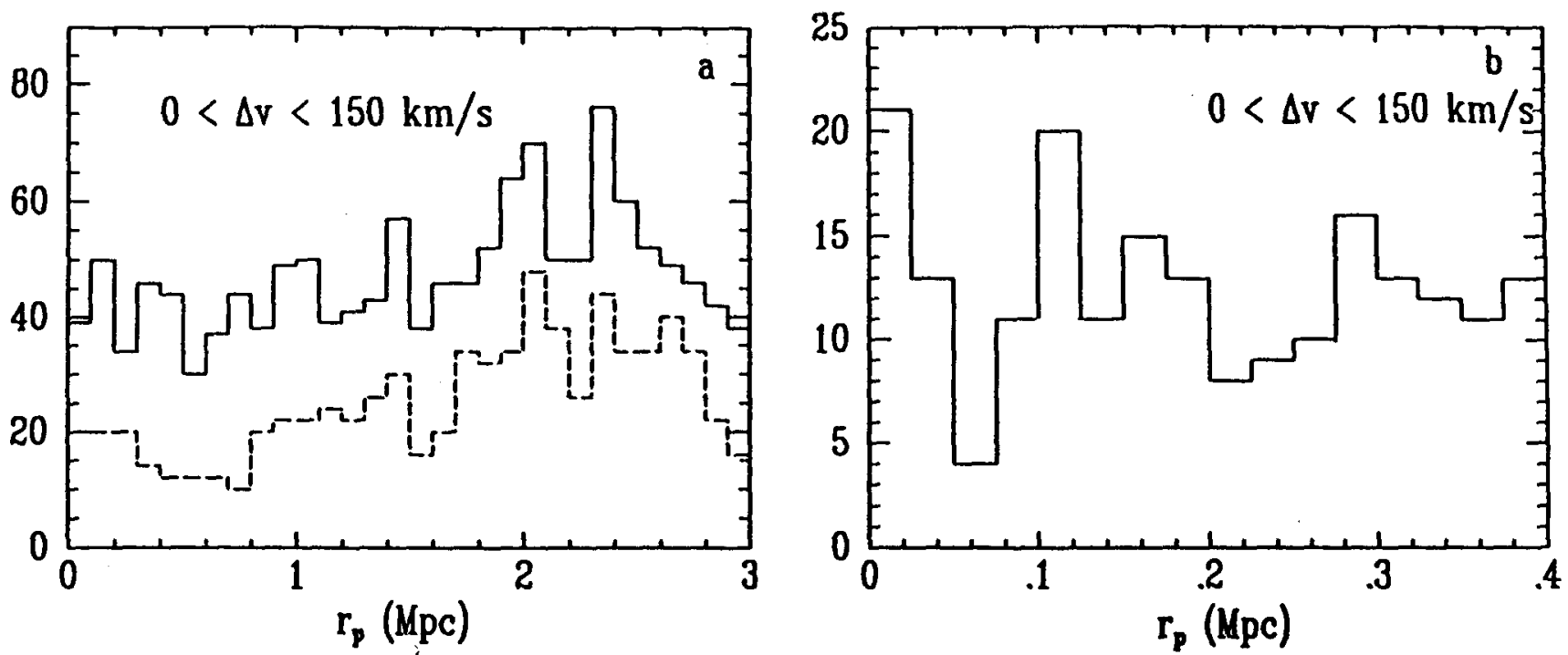

Figure 2: The projected separation $r_{p}$ distribution is given for low density pairs with small $\Delta v(0<\Delta v<150 \mathrm{~km} / \mathrm{s})$. The solid and dotted curves are the full sample and isolated pairs respectively, as in Figure 1. In a, the range of $r_{p}$ from 0 to $3 \mathrm{Mpc}$ is examined, while $b$ is a more detailed representation of small values of $r_{p}$ for the same distribution. 


\section{Galaxy Merger Rate}

The present galaxy merger rate can be estimated, using $\dot{R}$ and the present distribution of $r_{p}$. For example, if $\dot{R}$ is constant $(\beta=0)$ the initial $r_{p}$ distribution is simply shifted toward smaller values, retaining its shape. (Results will be quantitatively different for non-zero values of $\beta$.) The number of pairs to merge within a Hubble time $t_{H}$ is then the number of pairs in the present $r_{p}$ distribution with $r_{p}<\dot{R} \times t_{H} \times \sqrt{3} / 2$, where $\sqrt{3} / 2$ is a geometric factor due to projection. This is equal to the total number of low density pairs to merge by the present, assuming that the now hidden portion of the initial distribution of $r_{p}$, that has been shifted to merger, was also flat. The merger rate is conveniently expressed in terms of the dimensionless ratio $\xi=\dot{R} / \Delta v_{3 D}$ where $\Delta v_{3 D}$ is the median three dimensional velocity difference between pairs. We estimate that $\Delta v_{3 D} \sim 150 \mathrm{~km} / \mathrm{s}$ for "real pairs" from Figure 1, and $\xi$ can be computed from N-body results for particular orbits. Thus pairs with $r_{p}<1.9 \xi \mathrm{Mpc}$ have merged by the present, resulting in $\sim 49 \xi \%$ of low density primaries as merger products. From simulations of the merger of two $N=250$ galaxies on orbits with eccentricities ranging from circular to radial, we extract typical values of $\xi$ of $0.2-0.5$, yielding roughly $10-25 \%$ of low density galaxies as merger products. In this sample $18 \%$ of low density primaries are observed to be elliptical or S0 galaxies.

\section{Further Work}

In this paper we have chosen to focus on pairs in low density regions, specifically their implications for galactic halo sizes and the galactic merger rate. This work is part of a larger study (Charlton and Salpeter 1990, in preparation) that will include a more detailed study of the above issues as well as a presentation of $r_{p}$ and $\Delta v$ distributions for pairs in high density regions. In addition, we have analyzed scatter diagrams of $r_{p}, \Delta v$, and the geometric mean pair luminosity $L_{g m}$, and find none of the correlations that would have been expected for Kepler orbits (e.g., $\Delta v \sim r_{p}^{-1}$ ). In fact, we see a puzzling tendency for larger luminosity pairs to have smaller $\Delta v$. We would like to acknowledge support from NSF grant AST 87-14475 at Cornell and NSF grant AST 88-22297 at Steward Observatory.

\section{References}

Carlberg, R. G. 1987, M. N. R. A. S., 199, 1159.

da Costa, L. N., Pellegrini, P. S., Sargent, W. L., Tonry, J., Davis, M., Meiksin, A., Latham, D. W., Menzies, J. W., and Coulson, I. A. 1988, Ap. J., 257, 423.

Davis, M., and Peebles, P. J. E. 1983, Ap. J., 267, 465.

Huchra, J., and Geller, M. 1982, Ap. J., 257, 423.

Kharachentsev, I. D. 1990, in Paired and Interacting Galaxies: I.A.U. Colloquium 124, ed. J. W. Sulentic, W. Keel, and C. Telesco (N.A.S.A.), in press, p. 3

Ostriker, J. P., and Turner, E. L. 1979, Ap. J., 234, 785.

Rivolo, A. R., and Yahil, A. 1981, Ap. J., 251, 477.

Turner, E. L. 1976, Ap. J., 208, 20.

White, S. D. M. 1978, M. N. R. A. S., 184, 185. 